\title{
SAÚDE VOCAL E MHEALTH: NOVAS ALTERNATIVAS PARA ANTIGOS CENÁRIOS
}

\section{Vocal health and mHealth: novel alternatives for old scenarios}

\author{
Christina Cesar Praça Brasil \\ Universidade de Fortaleza - UNIFOR - Fortaleza (CE) - Brasil
}

\section{Daniele de Araújo Oliveira Carlos}

Universidade de Fortaleza - UNIFOR - Fortaleza (CE) - Brasil

\section{José Eurico Vasconcelos Filho}

Universidade de Fortaleza - UNIFOR - Fortaleza (CE) - Brasil

O mundo vivencia a era da comunicação, e a saúde é uma das áreas beneficiadas pela inserção de novos recursos na vida das pessoas. Nesse contexto, as Tecnologias da Informação e Comunicação (TICs) ocupam lugar de destaque, aplicandose ao processamento, armazenamento, busca e transmissão de informação em formato digital, trazendo mais velocidade e confiabilidade à troca de informações ${ }^{(1,2)}$. $\mathrm{Na}$ área da saúde, as TICs exercem um papel cada vez mais relevante, estando presentes nas áreas clínica, gerencial, assistencial e de apoio à decisão dos profissionais e gestores.

Nesse cenário, a tecnologia pode influenciar e modificar o modo de viver e de agir das pessoas, inclusive quando essas questões passam pelo contexto da promoção da saúde e do cuidado. Confirma-se, dessa forma, a relevância da incorporação das tecnologias na assistência à saúde ${ }^{(3,4)}$. No caso da saúde vocal, implica em um redimensionamento do autocuidado, uma vez que os acometimentos do aparelho fonador fazem parte do universo dos profissionais que utilizam a voz, destacando-se os professores, sendo uma realidade que requer enfrentamento por meio de medidas políticas e tecnológicas que ofereçam resolubilidade para esse problema tão recorrente.

Um estudo $^{(5)}$ realizado com 351 professoras de escolas municipais do ensino fundamental de Fortaleza, Ceará, mostrou que mais da metade delas apresenta mais de seis sintomas vocais, e que, mesmo considerando-se expostas a mais de seis fatores de risco vocal, nunca participaram de programa de saúde vocal, o que converge com outros estudos brasileiros ${ }^{(6,7)}$.

Ressalta-se que, além da escassez de ações educativas em saúde vocal, as políticas públicas brasileiras e a legislação voltadas à saúde do professor são falhas e restritas ${ }^{(8)}$, e, por isso, chama-se atenção para a importância do desenvolvimento de campanhas de comunicação em saúde e em recursos tecnológicos voltados à saúde vocal para reforçar a necessidade de cuidar continuamente da voz.

Diante desses dados, acredita-se que a ampliação do conhecimento sobre voz e a utilização de novas estratégias de comunicação possam favorecer o desenvolvimento de um vantajoso trabalho que contribua para a aprendizagem e o bem-estar de cada profissional da voz. Para isso, pode-se lançar mão de diversas tecnologias, dentre as quais se destacam as tecnologias móveis aplicadas à saúde (mHealth).

A área de pesquisa em saúde móvel ( $m$ Health), ramo da saúde eletrônica (eHealth) definido como "o uso de tecnologias de computação e comunicações móveis em cuidados de saúde e de saúde pública”, tem estado em constante expansão. As aplicações móveis para a saúde atendem a um público heterogêneo - médicos, enfermeiros, pacientes, cuidadores e pessoas saudáveis $^{(9)}$ - e a fins variados ${ }^{(10)}$, tais como informações nas diversas áreas da saúde, adesão a tratamento(s) e gestão da doença.

No Brasil, existem poucos estudos que abordam a temática saúde vocal e tecnologia. Contudo, é possível observar a inserção de várias ferramentas no cotidiano dos fonoaudiólogos, as quais lhes dão suporte e são reconhecidas como tipos de tecnologia. Podem ser citados como exemplo cartilhas, softwares, aplicativos informativos e plataformas de ensino a distância, todos voltados à saúde vocal, os quais facilitam o trabalho fonoaudiológico, porém não oferecem interatividade.

A partir dos recursos mencionados, acredita-se que a tecnologia $m$ Health, diante do dinamismo e das facilidades que oferece, como a possibilidade de realizar ações a qualquer tempo e em qualquer lugar, contribua para a promoção da saúde vocal dos professores, uma vez que as múltiplas atribuições e a falta de tempo levam o professor, muitas vezes, a menosprezar os seus problemas, buscando ajuda somente quando a(s) alteração(ões) vocal(is) já está(ão) instalada(s) ${ }^{(11)}$.

Ante a carência de aplicativos que favoreçam os cuidados com a saúde vocal, desenvolveu-se, na Universidade de Fortaleza, por meio de uma parceria entre o Programa de Pós-Graduação em Saúde Coletiva e o Laboratório de Inovação e 
Novos Negócios do Núcleo de Aplicação em Tecnologia da Informação (NATI), o aplicativo VoiceGuard, uma ferramenta que propicia o apoio individualizado na gestão do uso da voz. Esse aplicativo apresenta como diferencial a oferta de uma variedade de recursos para o gerenciamento e o monitoramento amplo e integral da saúde vocal, inclusive em tempo real, favorecendo maior autonomia do professor e a redução dos acometimentos vocais. Acredita-se que essa tecnologia seja uma importante ferramenta para a promoção e o cuidado com a saúde vocal, sendo um recurso moderno para o enfrentamento de um antigo problema: as alterações vocais dos professores.

A Revista Brasileira em Promoção da Saúde acredita na disseminação desse e de outros inovadores produtos tecnológicos aplicados à saúde e ao bem-estar, dada a velocidade das interações científicas em prol do acesso ilimitado à saúde.

\section{REFERÊNCIAS}

1. Carr NG. Does it matter? Information technology and the corrosion of competitive advantage. Boston: Harvard Business Press; 2004.

2. Costa SRS, Duqueviz BC, Pedroza RLS. Tecnologias digitais como instrumentos mediadores da aprendizagem dos nativos digitais. Psicol Esc Educ. 2015;19(3):603-10.

3. Silva RC, Ferreira MA. A tecnologia em saúde: uma perspectiva psicossociológica aplicada ao cuidado de Enfermagem. Esc Anna Nery Rev Enferm. 2009;13(1):169-73.

4. Pereira RB, Coelho MA, Bachion MM. Tecnologias de informação e registro do processo de enfermagem: estudo de caso em UTI neonatal. Rev Eletrônica Enferm [Internet]. 2016 [acesso em 2016 Nov 20];18:1-13. Disponível em: https://www. revistas.ufg.br/fen/article/view/35135

5. Brasil CCP. A voz da professora não pode calar: sentidos, ações e interpretações no contexto da integralidade em saúde [tese]. Fortaleza: Universidade Estadual do Ceará, Universidade Federal do Ceará e Universidade de Fortaleza - Associação Ampla; 2015.

6. Behlau M, Zambon F, Guerrieri AC, Roy N. Epidemiology of voice disorders in teachers and nonteachers in Brazil: prevalence and adverse effects. J Voice. 2012;26(5):665.e9-18.

7. Pascotini FS, Ribeiro VV, Cielo CA. Voz de professoras do ensino fundamental com queixas vocais de diferentes redes de ensino. Distúrbios Comun. 2015;27(1):138-50.

8. Ferreira LP, Servilha EAM, Masson MLV, Reinaldi MBFM. Políticas públicas e voz do professor: caracterização das leis brasileiras. Rev Soc Bras Fonoaudiol. 2009;14(1):1-7.

9. Free C, Phillips G, Felix L, Galli L, Patel V, Edwards P. The effectiveness of M-health technologies for improving health and health services: a systematic review protocol. BMC Res Notes. 2010;14(5):2-7.

10. Riley WT, Rivera DE, Atienza AA, Nielsen W, Allison SM, Mermelstein R. Health behavior models in the age of mobile interventions: are our theories up to the task? Transl Behav Med. 2011;1(1):5371.

11. Cielo CA, Portalete CR, Bastilha GR, Ribeiro VV. Perfil vocal, ocupacional e de saúde geral de docentes de Santa Maria/ RS. Rev CEFAC. 2016;18(3):635-48.

\section{Endereço para correspondência:}

Christina Cesar Praça Brasil

Universidade de Fortaleza - UNIFOR

Programa de Pós-graduação em Saúde Coletiva

Avenida Washington Soares, 1321

Bairro: Edson Queiroz

CEP: 60811-905 - Fortaleza - CE - Brasil

E-mail: cpraca@unifor.br 